\title{
Ameliorative Effect of Pentoxifylline a Phosphodiesterase-5 on Acrolein Induced Oxidative Damage
}

\author{
Jamshid Karimi, ${ }^{1}$ Heidar Tavilani, ${ }^{1}$ and Akram Ranjbar ${ }^{2, *}$ \\ ${ }^{1}$ Department of Biochemistry, Hamadan University of Medical Sciences, Hamadan, IR Iran \\ ${ }^{2}$ Department of Toxicology and Pharmacology, Faculty of Pharmacy, Hamadan University of Medical Sciences, Hamadan, IR Iran \\ *Corresponding author: Akram Ranjbar, Department of Toxicology and Pharmacology, Faculty of Pharmacy, Hamadan University of Medical Sciences, Hamadan, IR Iran. \\ E-mail: akranjbar1389@yahoo.com
}

Received: March 9, 2014; Accepted: May 12, 2014

\begin{abstract}
Background: Pentoxifylline(PTX), a methylxanthine derivative and nonspecific type 5 phosphodiesterase(PDE)inhibitor, is a drug widely used in the management of peripheral arterial disease.

Objectives: The aim of this study was to investigate the possible protective role of PTX on toxicity of acrolein (ACR).

Materials and Methods: In this experimental study, 30 male rats were equally divided in to 6 groups ( 5 rats each). Group I (control) that received normal saline, group II was given ACR ( $2 \mathrm{mg} / \mathrm{kg} /$ day, i.p.). Animals of group III received only PTX (50 mg/kg/day, i.p.). Group IV was given ACR + PTX, groups V received only vitamin E (15 mg/kg/day, s.c.) and group VI was given vitamin E combination to ACR once daily for 14 dayes. Oxidative damage were measured by oxidative biomarkers such as glutathione peroxidase (GPX), superoxide dismutase (SOD), lipid peroxidation (LPO) and total glutathione (GSH) in blood of rats.

Results: At the end of the experiment, the plasma of the animals was separated. In the blood plasma, ACR reduced total glutathione(GSH), SOD and GPX compared control group. Also, the LPO was increased in the ACR group as compared with controls. PTX ameliorated LPO, SOD and GPx in blood of ACR-induced changes.

Conclusions: These findings suggest that PTX may provide a promising approach for the treatment of ACR-related diseases throughout reduction oxidative injuries.

Keywords: Oxidative stress; Pentoxifylline; Acrolein
\end{abstract}

\section{Background}

Acrolein (ACR) is a highly electrophilic alpha, beta-unsaturated aldehyde to which humans are exposed in many situations, such as alcoholic beverages, water and foods such as cheese, donuts and coffee, and is also formed during combustion of organic materials, including engine exhaust, wood, tobacco and over-heated cooking oils [1]. Notably, ACR is also produced endogenously by normal cellular metabolism. ACR can be formed in various tissues via lipid peroxidation (LPO), metabolism of $\alpha$-hydroxyamino acids, polyamines oxidation and via metabolism of drugs, such as the anticancer drug cyclophosphamide [2,3].

ACR is a strong and highly reactive electrophile and remains active in the body for several days [4]. Also, ACR contributes directly to cellular oxidative stress [5]. Pentoxifylline (PTX), a methylxanthine derivative and nonspecific type 5 phosphodiesterase inhibitor, is a drug that widely used in the management of peripheral arterial disease and, in particular, for intermittent claudication [6]. The mechanism underlying its beneficial effects appears to be related to the improvement of cellular functions and modifications in the plasma that improve microcirculatory perfusion in both peripheral and cerebral vascular beds [7]. In recent years, in vitro and in vivo experiments indicat- ed an additional therapeutic potential for PTX as an antiinflammatory, immunomodulator agent and antioxidative properties $[8,9]$. However, the antioxidative effects of PTX against ACR-induced oxidative damage as well as remain unknown.

\section{Objectives}

Here we investigated the effects and mechanisms of PTX on ACR-induced oxidative damage in blood in male rat.

\section{Materials and Methods}

In this experimental study, 30 adult male Wistar rats weighing 180 - $250 \mathrm{~g}$ maintained on a 12 hour light/dark cycle with free access to tap water and standard laboratory chow were used. Animals were randomly divided into 6 groups and treated for 2 weeks i.p. The groups ( 5 rats each) were as follows: control group, ACR group, PTX group, PTX + ACR group, alpha-tocopherol acetate (AT) group, and ACR + AT group.

ACR was administered (2 $\mathrm{mg} / \mathrm{kg} /$ day, i.p.) alone or in combination with PTX (50 mg/kg/day, i.p.), and AT as (15 $\mathrm{mg} / \mathrm{kg} /$ day, s.c.). One group of animals received only nor- 
mal saline and was assigned as control. At the end of the treatment, 24 hours after last dose of treatment, animals were killed, and then blood samples were collected from heart in tubes and serum was isolated.

Malondialdehyde (MDA), 2-thiobarbituric acid (TBA), trichloroacetic acid (TCA), n-butanol, sucrose, ethylenediamine tetraacetic acid (EDTA), glutathione peroxidase (GPx) and superoxide dismutase (SOD) (Ransel kit, Randox Laboratories Ltd, Crumlin, UK), bioxytech GSH kit (Oxis Research, USA), were used in this study. All other chemicals were obtained from the Sigma Company.

The lipid peroxidation (LPO) product in tissues was determined by TBA reagent, expressed as the extent of malondialdehyde (MDA) productions during an acid heating reaction. Briefly, the samples were diluted by $1.5 \mathrm{~mL}$ TCA $(20 \%$ $\mathrm{w} / \mathrm{v}$ ) was added to $250 \mu \mathrm{L}$ of this samples and centrifuged in $3000 \mathrm{~g}$ for 10 minutes. Then, the precipitation was dissolved in sulfuric acid and $1.5 \mathrm{~mL}$ of the mixture was added to $1.5 \mathrm{~mL}$ of TBA $(0.2 \% \mathrm{w} / \mathrm{v})$. The mixture was then incubated for $1 \mathrm{~h}$ in a boiling water bath. Following incubation, $2 \mathrm{~mL}$ of n-butanol was added, the solution centrifuged, cooled and the absorption of the supernatant was recorded in 532 $\mathrm{nm}$. The calibration curve of tetraethoxypropane standard solutions was used to determine the concentrations of TBA + MDA adducts in samples [10].

Level of total glutathione (GSH) was measured using colorimetric assay kit. The kit uses 5, 50-dithiobis-2-nitrobenzoic acid (DTNB) and glutathione reductase. The procedure was followed according to manufacturer's instruction and the levels were quantitated as micromolar GSH based on standard supplied along with the kit.

The activity of $\mathrm{Cu} / \mathrm{Zn}$ SOD was measured using a commercial kit (Ransod kit, Randox Laboratories Ltd, Crumlin, UK). Measurement of the enzyme was based on the generation of superoxide radicals produced by xanthine and xanthine oxidase and reacted with 2-(4-iodophenyl)-3-(4-nitrofenol) 5-phenyltetrazolium chloride (INT) to form a red formazan dye. The formazan was read at $505 \mathrm{~nm}$. One unit of $\mathrm{Cu} /$ Zn SOD was defined as the amount of enzyme necessary to produce $50 \%$ inhibition in the INT reduction rate.

The amount of GPx was determined using a commercially available kit (Ransel kit, Randox Laboratories Ltd, Crumlin, UK) by measuring the rate of oxidation of NADPH at $340 \mathrm{~nm}$. A unit of enzyme was expressed as the amount of enzyme needed to oxidize $1 \mathrm{nM}$ of NADPH oxidase/minute.

The protein content was quantified by the method of Bradford.Concentrated Coomassie blue (G250) was diluted in $250 \mu \mathrm{L}$ distilled water, and then $750 \mu \mathrm{L}$ of this diluted dye was added to $50 \mu \mathrm{L}$ of sample. The mixture was incubated at room temperature for 10 minutes and an absorbance measurement was taken at $595 \mathrm{~nm}$ by a spectrophotometer. A standard curve was constructed by using bovine serum albumin ranging between 0.25 and $1 \mathrm{mg} / \mathrm{mL}$ [11].

All data were analyzed with SPSS-18 employing oneway ANOVA followed by Tukey post hoc test. Data are expressed as Mean \pm SEM. P value $<0.05$ was taken as statistically significant.

\section{Results}

In blood: ACR caused a significant increase in LPO when compared to control $(\mathrm{P}=0.02)$. PTX caused a significant decrease in LPO when compared to ACR group $(P=0.03)$. Coadministration of PTX with ACR significantly reduced ACR induced LPO $(P=0.05)$. AT reduced LPO when compared to ACR $(P=0.01)$. Coadministration of AT with ACR significantly reduced ACR induced LPO ( $\mathrm{P}=0.02$, Figure 1$)$.

In blood: ACR caused a significant decrease in SOD activity when compared to control $(\mathrm{P}=0.04)$. PTX caused a significant increase in SOD activity when compared to ACR group. AT induced SOD activity when compared to $\operatorname{ACR}(P=0.03$, Figure 2$)$.

In blood: ACR caused a significant decrease in GPx activity when compared to control $(\mathrm{P}=0.02)$. AT induced GPx activity when compared to ACR $(P=0.01$, Figure 3$)$.

In plasma: Administration of ACR decreased GSH in comparison to controls $(\mathrm{P}=0.04)$. Treatment with PTX increased GSH as compared to ACR group $(\mathrm{P}=0.01)$. AT significantly increased GSH level as compared to ACR group $(P=0.04$, Figure 4$)$.

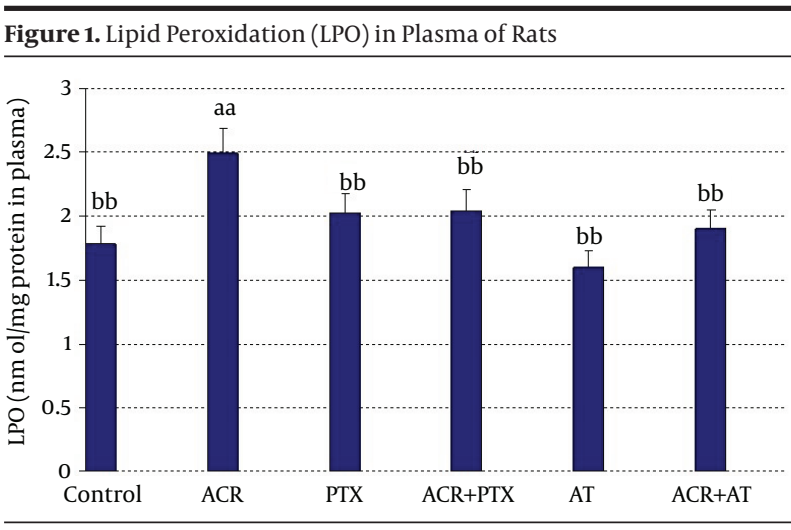

aa:Significantly different from control group at $\mathrm{P}<0.05$. bb: Significantly different from ACR group at $\mathrm{P}<0.05$. ACR, acrolein; PTX, Pentoxifylline; AT, (alpha-tocopherol; vitamin E).

Figure 2. Superoxide Dismutase (SOD) Activity in Plasma of Rats

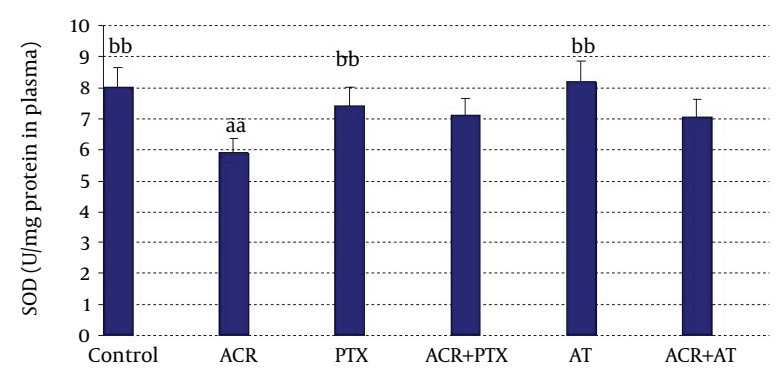

aa: Significantly different from control group at $\mathrm{P}<0.05$. bb: Significantly different from ACR group at $\mathrm{P}<0.05$. ACR, acrolein; PTX, Pentoxifylline; AT, (alpha-tocopherol; vitamin E). 


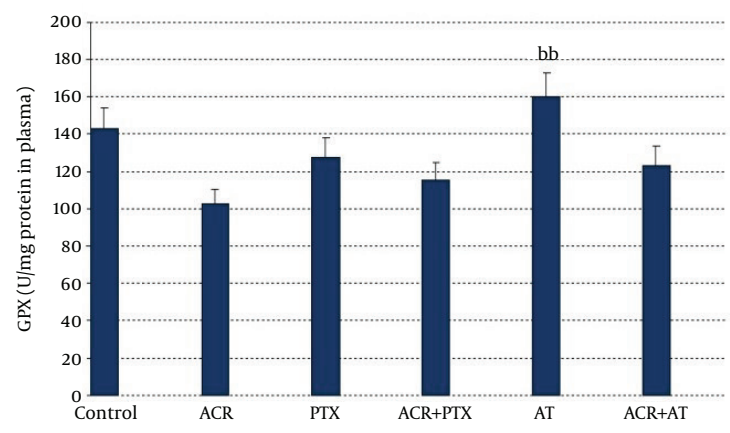

aa: Significantly different from control group at $\mathrm{P}<0.05$. bb: Significantly different from ACR group at $\mathrm{P}<0.05$. ACR, acrolein; PTX, Pentoxifylline; AT, (alpha-tocopherol; vitamin E).

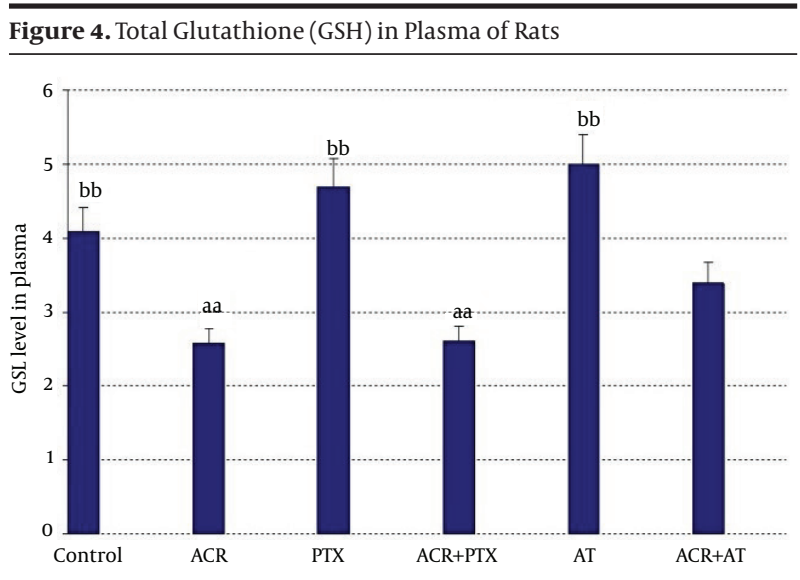

aa: Significantly different from control group at $\mathrm{P}<0.05$. bb: Significantly different from ACR group at $\mathrm{P}<0.05$. ACR, acrolein; PTX, Pentoxifylline; AT, (alpha-tocopherol; vitamin E).

\section{Discussion}

Treatment of rats with PTX on ACR-induction oxidative stress attenuated subsequent oxidative damage. In the present study, our intention was to address a novel link between the protective roles of PTX towards oxidative stress caused by ACR in blood. The adverse effects of ACR on human health are relevant since ACR is a ubiquitous pollutant present in the environmental, food and water, and human exposures are common. Being an endogenous toxin, ACR is especially insidious [12]. Previously, other researchers had demonstrated that ACR-induced oxidative damage is associated with neurodegenerative diseases, especially with Alzheimer's disease (AD) in vitro system [13]. ACR is primarily metabolized via rapid reaction with sulfhydryl groups of glutathione forming mercapturic acid; this is ultimately eliminated in the urine [14]. Thus, ACR contributes directly to cellular oxidative stress via loss of glutathione [5]. Our re- sults showed that ACR reduced GSH concentration in blood. But it was significantly improved with PTX in this sample. Moreover, ACR can easily move across cell membranes and tissues due to its solubility in water and alcohol, and hence, high concentrations of ACR produced by LPO to damage cells. Notably, in vivo, LPO also results in the formation of aldehydes by-products, including ACR, malondialdehyde (MDA), and 4-hydroxynonenal (4$\mathrm{HNE}$ ). Among them, ACR is the most reactive and the formation of ACR is earlier than 4-HNE $[15,16]$. In the present study, ACR treatment caused significant increase in LPO in blood and liver mitochondrial cell.

Also, previous studies have shown that both GPx and SOD abrogated reactive oxygen species (ROS) generation [9, $17,18]$. In addition, this result was indicated reporting decreased activities of SOD and GPx in ACR group compared with control. PTX is a cAMP phosphodiesterase inhibitor [19]. Administration of PTX leads to increased intracellular cAMP accumulation in several cell types, including mononuclear phagocytes, microglia, neutrophils, vascular smooth muscle, and endothelium [20]. Increased intracellular cAMP affects gene transcription through several transcription factors, such as cAMP response element-binding protein. Multiple complementary anti-inflammatory effects of PTX could be responsible for its neuroprotective efficacy, including inhibition of neutrophil or monocyte/ microglial activation, attenuation of inflammatory mediator production (e.g. platelet-activating factor or TNF-a, and prevention of endothelial-leukocyte adhesion [19-22]. Other effects of PTX, independent of its antioxidative actions, could also contribute to its. Thus PTX might be suitable for clinical trial as a human oxidative induced by ACR.

Therefore, AT mainly acts as a chain-breaking antioxidant and radical scavenger, protecting cell membrane against oxidative damage [23]. In addition, AT also regulates production of ROS and maintains oxidative phosphorylation in the mitochondria and accelerates compensation of high-energy metabolites [24, 25]. The present results showed AT ameliorated oxidative induced ACR damages in blood. Taken together, to the best of our knowledge, we have provided evidence for PTX can prevent ACR induced oxidative injury and also restore that same AT.

\section{Acknowledgements}

This study was supported by a grant from Vice Chancellor of Research of Hamadan University of Medical Sciences. This research article is taken from the research code 4750 written up by Dr. Ranjbar.

\section{Authors' Contributions}

All authors had equal role in design, work, statistical analysis and manuscript writing.

\section{Funding/Support}

Hamadan University of Medical Sciences. 


\section{References}

1. Uchida K, Kanematsu M, Sakai K, Matsuda T, Hattori N, Mizuno Y, et al. Protein-bound acrolein: potential markers for oxidative stress. Proc Natl Acad Sci U S A.1998;95(9):4882-7.

2. Uchida K. Current status of acrolein as a lipid peroxidation product. Trends Cardiovasc Med.1999;9(5):109-13.

3. Kehrer JP, Biswal SS. The molecular effects of acrolein. Toxicol Sci. 2000;57(1):6-15.

4. Ghilarducci DP, Tjeerdema RS. Fate and effects of acrolein. Rev Environ Contam Toxicol.1995;144:95-146.

5. Pocernich CB, Cardin AL, Racine CL, Lauderback CM, Butterfield DA. Glutathione elevation and its protective role in acrolein-induced protein damage in synaptosomal membranes: relevance to brain lipid peroxidation in neurodegenerative disease. Neurochem Int. 2001;39(2):141-9.

6. Banfi C, Sironi L, De Simoni G, Gelosa P, Barcella S, Perego C, et al Pentoxifylline prevents spontaneous brain ischemia in strokeprone rats.J Pharmacol Exp Ther. 2004;310(3):890-5.

7. Strutz F, Heeg M, Kochsiek T, Siemers G, Zeisberg M, Muller GA. Effects of pentoxifylline, pentifylline and gamma-interferon on proliferation, differentiation, and matrix synthesis of human renal fibroblasts. Nephrol Dial Transplant. 2000;15(10):1535-46.

8. Bruno Rde B, Marques TF, Batista TM, Lima JC, de Arruda KG, Lima $\mathrm{PF}$, et al. Pentoxifylline treatment improves neurological and neurochemical deficits in rats subjected to transient brain ischemia. Brain Res. 2009;1260:55-64.

9. Ranjbar A, Ghahremani MH, Sharifzadeh M, Golestani A, GhaziKhansari M, Baeeri M, et al. Protection by pentoxifylline of malathion-induced toxic stress and mitochondrial damage in rat brain. Hum Exp Toxicol.2010;29(10):851-64.

10. Ernster L, Nordenbrand K. [92a] Microsomal lipid peroxidation. 1967;10:574-80.

11. Bradford MM. A rapid and sensitive method for the quantitation of microgram quantities of protein utilizing the principle of protein-dye binding. Anal Biochem. 1976;72:248-54

12. Beauchamp RJ, Andjelkovich DA, Kligerman AD, Morgan KT, Heck HD. A critical review of the literature on acrolein toxicity. Crit Rev Toxicol.1985;14(4):309-80.

13. Bradley MA, Markesbery WR, Lovell MA. Increased levels of 4-hy- droxynonenal and acrolein in the brain in preclinical Alzheimer disease. Free Radic Biol Med. 2010;48(12):1570-6.

14. Adams JJ, Klaidman LK. Acrolein-induced oxygen radical formation. Free Radic Biol Med.1993;15(2):187-93.

15. Jaeschke $\mathrm{H}$, Kleinwaechter C, Wendel A. The role of acrolein in allyl alcohol-induced lipid peroxidation and liver cell damage in mice. Biochem Pharmacol. 1987;36(1):51-7.

16. Custovic Z, Zarkovic K, Cindric M, Cipak A, Jurkovic I, Sonicki Z, et al. Lipid peroxidation product acrolein as a predictive biomarker of prostate carcinoma relapse after radical surgery. Free Radic Res. 2010;44(5):497-504.

17. Rathore N, John S, Kale M, Bhatnagar D. Lipid peroxidation and antioxidant enzymes in isoproterenol induced oxidative stress in rat tissues. Pharmacol Res.1998;38(4):297-303.

18. Mittler R. Oxidative stress, antioxidants and stress tolerance. Trends Plant Sci. 2002;7(9):405-10.

19. Bessler H, Gilgal R, Djaldetti M, Zahavi I. Effect of pentoxifylline on the phagocytic activity, cAMP levels, and superoxide anion production by monocytes and polymorphonuclear cells. J Leukoc Biol. 1986;40(6):747-54.

20. Zein CO, Lopez R, Fu X, Kirwan JP, Yerian LM, McCullough AJ, et al. Pentoxifylline decreases oxidized lipid products in nonalcoholic steatohepatitis: new evidence on the potential therapeutic mechanism. Hepatology. 2012;56(4):1291-9.

21. Zein CO, Yerian LM, Gogate P, Lopez R, Kirwan JP, Feldstein AE, et al. Pentoxifylline improves nonalcoholic steatohepatitis: a randomized placebo-controlled trial. Hepatology. 2011;54(5):1610-9.

22. Vakili A, Mojarrad S, Akhavan MM, Rashidy-Pour A. Pentoxifylline attenuates TNF-alpha protein levels and brain edema following temporary focal cerebral ischemia in rats. Brain Res. 2011;1377:119-25.

23. Pazdro R, Burgess JR. The role of vitamin E and oxidative stress in diabetes complications. Mech Ageing Dev. 2010;131(4):276-86.

24. Mao G, Kraus GA, Kim I, Spurlock ME, Bailey TB, Zhang Q, et al A mitochondria-targeted vitamin $\mathrm{E}$ derivative decreases hepatic oxidative stress and inhibits fat deposition in mice. J Nutr. 2010;140(8):1425-31.

25. Ranjbar A. Evidence of oxidative damage in paraquat toxicity [In Press]. Zah J Res Med Sci. 2015. 